Physica D 38 (1989) 377-383

North-Holland. Amsterdam

\title{
HUILL-GENERATING WALKS
}

\author{
Robert M. ZIFF

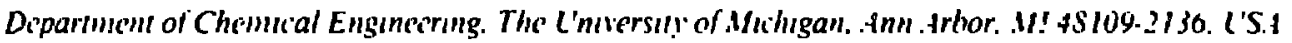

\begin{abstract}
A hull-generating walk (HGW) is a lype of kinetıc random walk that generates the hull or perımeler of a percolation cluster. and thus has a fractal dimension of 1.75 . Some examples of HGW's for site and bond percolation on a square lattice are desiribes.
\end{abstract}

\section{Introduction}

A percolation cluster is a collection of occupied sites connected to each other by paths along neqrestneighbor pairs of siles, and surrounded inside and outsife by va'ant sites. (For bond percolation. this defir.tion holds by reformulating the problem as site percolation on the coverıng 'suice.) A closed circuit along the boundary of adiacent ocrupted and vacani sites is called a perimeter or the hull of the cluster. The term "hull" was first used by Mandelbrot [1] to describe the island of points enclosed by the external brundary of a cluster. but it has been gene, alized to refer :o the boundary as well, and that meaning will be used here. One can have both external hulls, in which the occupied sites are on the inside and the vacant sites on the outside, and internal hulls, in which the occupied sites are on the outs:cie and the vacant sites are on the inside.

Mandelbrot's influcnce on the study of percolation hulls goes far beyond the coining of the name, of course. The invention of Iractals and the resulting inter :st in the study of growth processes and geometric propertues has sumuiated a greal deal of work on percolation clusters and their hulls. which are among the simplest and most clegant of ranüura tractats. ama which result from many growth and epidemic models iset for example refs. [2-4]). While perimeters of percolation clusters have been sludied for many years in the contcit of cluster siaustics [5.6] or boundary properies [7]. the introducion of tractals has led to

Esses an horiour of Benoil B. Miandelbror

Fractals in Phusles - A. tharon! and J Feder (cditors; substantual advances in the understanding of their properties.

Indeed, the fractal nature turned out to be the kes to the discovery of express ons for all the rritical exponents of percolation hulls. In their investigation of the scaling of percolation-gradient frontters (hulls). Sapcval. Rosso and Gouyet [8] were led to the conjecture that the fractal dimension of the hull is evactly

$P=i+1 i t=1.75$. 11:

" here $l^{\prime}=-{ }^{\prime}$ is the correlauon-length exponent. This conkcture s suppored by numerical siudies $\mid S-11)$ Then. by sialıg arguments. (1) was show 3 /10 :2]

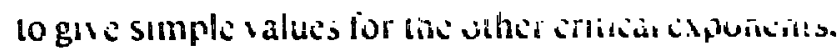
such as $y^{\prime}=2$ (for the mean hull-size exponens) and $\beta^{\prime}=1 / 3$. Finally. Saleur and Duplantier $[13 j$ derived evact expressions for these zxponents from tirst principles. thus verifying (1) by scaling. Theoretical arg' 'ments for ( $i$ ) have also been given by Bunde and Gouye [14]. This work has shown that periolation hulls have simpler critical euponents than the clusters themselves.

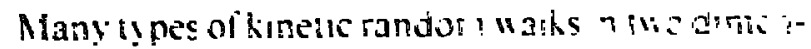

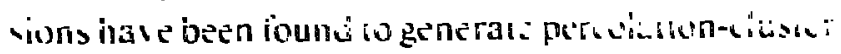

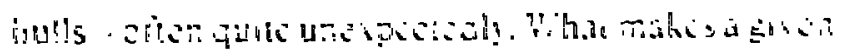

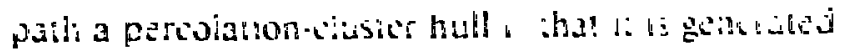
with the same probabiling " weght! as it would be found on a laulce that has teen randomls populatid with oicupled and vacant sites. In this paper I am concerned with these walks. which I call hull-generating walks, HGils:

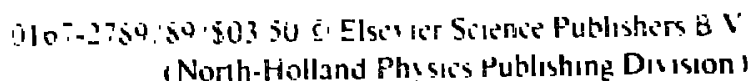


In general, HGWs ve the following pruperties:

(1) They are generated on a latice that starts out completely blank (untasted). ex:ept perhaps for one or two sites to stari the walk.

(2) They leave a path of both occupied and vacant sites, or some equivalent representation of a cluster boundary.

(3) Their growth process is local (depending only upon a local set of sites) and kinetic (they grow step by step ).

(4) They eventually close to form a completed loop (on an infinite lattice).

(5) Once thay closs, they are no longer kunetic in nature, meaning that their probability of growiug (weight) is no longer dependent upon th: starting foint.

(6) When closed, their weight is that of the corresponding perimeter of a percolation clister. If the occupied sites are on the outside, then the path represents an e.ternal perimeter, while if the occupied sites are in the inside then the path represents an internal perimeter.

(7) Their fractal dimension is 1.75 .

HGW's wcre first introduced b) Ziff. Cummings. and Stell [15.16] for the specific purpose of generaling percolation clusier perimeters. They were independently found to result from quite diffarenı consideranons. Kremer and Lyklema [17] devised an indetin:tely growing self-avoiding walk (IGSAW) on a square lattic which satislies properties (1)-(j) above. but not the rest because the walk; never close. How ever. Weinrib and Trugman [12] siudied a sımilar walk on a honeycomb lattice. which they call the smart kinetic walk (SKW), and found that it is precisely a HGW for site percolation on the dual (tr1angular) lattice. Gunn and Ortuño [18.] considered a random system of sites on a lattice that have the pronerly of rolatıng the direction of a ualk pass!ng through them by given amounts. and found under cortan circumstances that the parhs are equit alent io a HGIV for bond percolation on the square lattice. A s.milar walh was used by Grassberger [11]. Recint!. Ruui es al. [ 19] have introduced a sien-b)sicp tiling process that is equis alent to the Gunil and
Ortuño model and also to the representation by Saleur and Duplantier [13], and thus is eciaivilent io the bond-percolation HGW. These models will be described in more detail below.

HGWs are useful for finding the percolation threshold [ 10,20$]$ : in fc.t using the gradient-probability inethod [20] they appear to be the most efficient Monte Carlo way to find $p_{c}$. HGWs allow one to generate the hull of the backbone of a percolation cluster [21], and also the "accessible perimeter" of Grossman and Aharony [22], as discussed below. Coniglio et al. [23] and later Duplantier and Saleur [24] and Bradley [25] have argued that the HGW is appropriate to represent a two-dimensional polymer chain at the $\theta$ or $\theta^{\circ}$ point, and so these walks are more than jusi a mathematical curiosity but have physical significance as well.

In general. a HGW can be constructed for a given system by the following procedure [15]: First devise an algcrithm to trace out the perimeter of an existing cit'ster. Then repeat the same algorithm on a blank (untested) lattice, with the modification that when the state of any site that is still untested is needed. that site is made "occupied" with probability $p$ and "vacant" otheru ise, and the algorithm is continued according to that decision. Moreover, the state of the slle must be remembered so that if it is ever visited again it will be treated the same way. The perimeter produced by this walk has the same weight as the corresponding perimetcr on a populated lattice, because, in randum percolation, the state of a site (or bond) is assigned with statistical independence, and it is irrele ant whether the choise of the state is made beforehand or during the walk.

An interesting aspect of the HGW' is the behavior when $\mu$ is increased beyond $p_{\mathrm{c}}$. For $\mu<p_{i}$. external hulls are more likely. while for $p>\mu_{i}$ the internal hulls are more like!! [!5]. How ever, the existence of the infinite cluster is not evident - there is no hull associated wi.h tt. The internal, ulls that are produced "hen $p>p_{i}$ my be holes within the infin : or holes within a 'arger finite cluster. There is a naturai symmetry for the behavior or the walks about $\mu_{2}$. "hich for site percolation on the triangular latlice and 
bc.ld percolation on the square lattice is perfect because of the identity of matching lattices.

By considering different lattices and definitions of the perimeter, a great variety of HGWs can be constructed, all of which satisfy properties $(1)-(i)$ above. Furthermore, even for the same system one can devise different walks that generate the perimeter. This will be illustrated by some e::amples of HGWs for site arid bond percolation os a square lattice.

\section{Site percolation on a square lattice}

In fig. I a simple cluster of five occupred sites
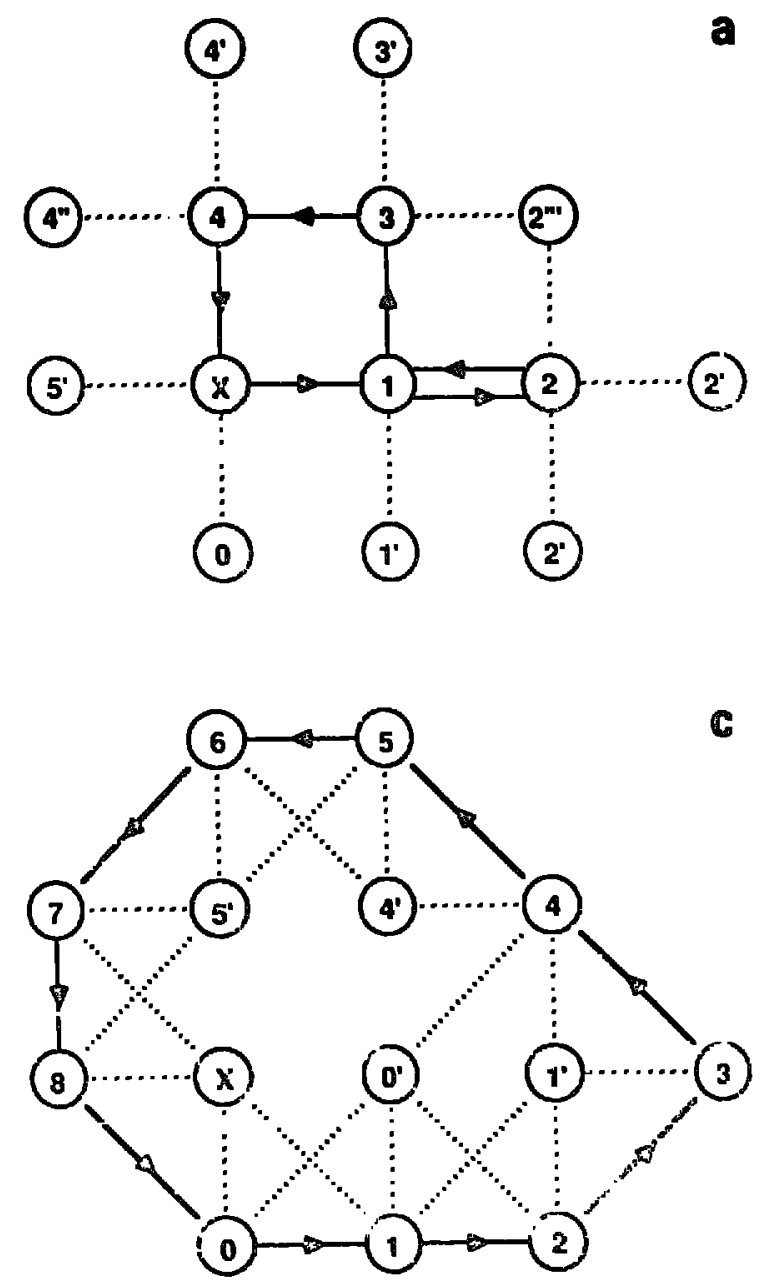

(shaded circles) anu' nine vacant sites (open circies) for site perrolation on a square lattice is shown. First consider the walk that follows tne occupied sites at the boundary. which is illustrated in fig. la. To follow that boundary, the walker moves from occupied site to occupied site. always keeping vacant sites to its right. The walker "looks" first to the right. then straight, then left, then back, where "straight" is the direction of the previous step. The HGW that results from this process follow's the following wile on a initially untested lattice: If the site being looked at is

(1) occupied: the walker moves to it:

(L) yacant: the walker looks to the next site in counter-clockwise order:

(3) untested: the site is made "occupied" with
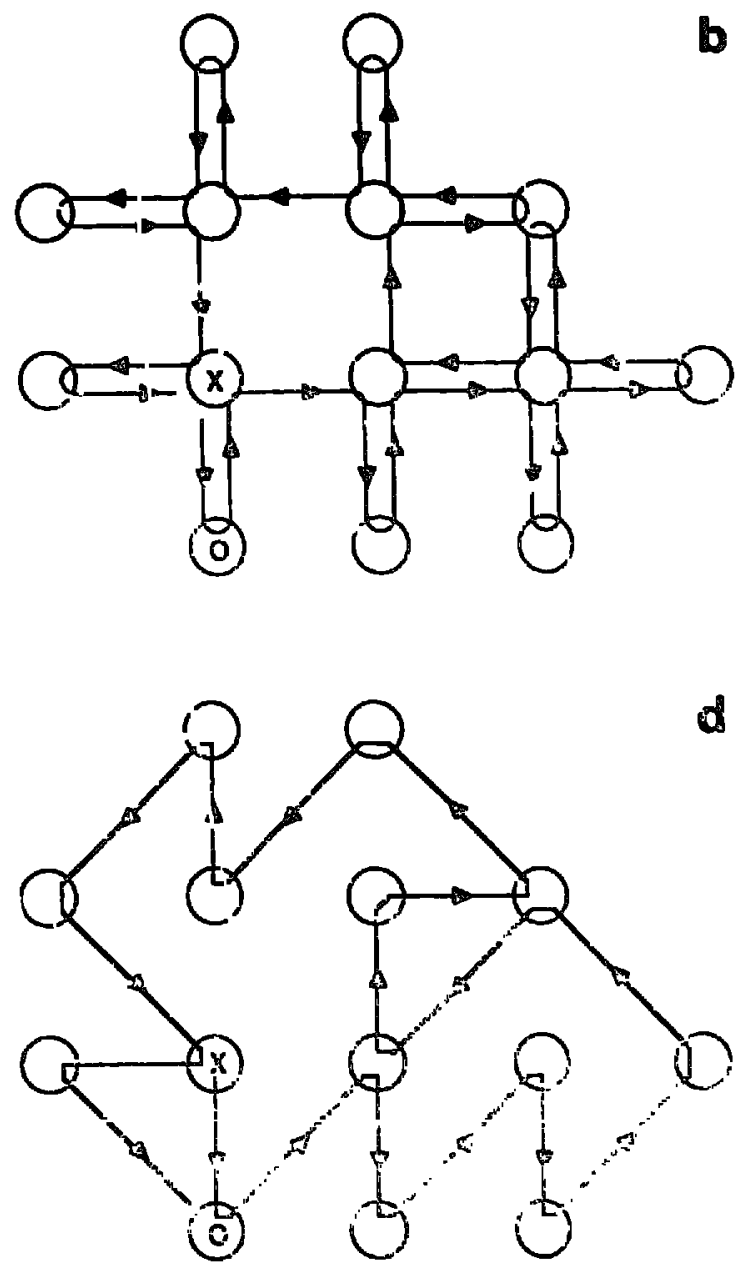

Fig. 1. Sit: percolation on a square lattice: (a) the walk that connects occupied siles (shaded circles) of the penmeter. (b) the eculsalent "blirid ant" walk. (c) the generic walk that moves along the vacant sites (open circles) of the p rnmeter. and (d) a neu more eiticieni walk that goes along diagonals from vacant stıes and verical and horizonıl lines from occupıed sites. 
probability $p$ and $(1)$ is followed. otherw ise the site is made "vacant" and ( 2 ) is followed.

The walk is started by placing down the occupiedvacant site pair (marked $X$ and $O$ in fig. $1 a$ ), and finishes when the walker returns to $X$ and $a_{n}$ tempts to go in the direction of the first step. Also in fig. la, the occupled sites are numbered according to the order in which they are created. and the vacant sites are labelled with a prime. double prime. etc.. and a number corresponding to the occupied site where the walker was when the vacant site was created. Thus after the walker reaches occupied site 1 , the vacant site $I^{\prime}$ is first created. before the occupied site 2 is created. Because of the three varant sites created around 2. the walker must backırack to site I, which is allowed here. When the walker reaches occupied site 3. il lirst looks to the right and sees 2" , which was already made vacant before, and so goes on to site $3^{\prime}$ - and so on. This walk was simulated very extensively in refs. [ 10.15.20]. where the scaling relations and (1) were serified, and the value of $r$ (square) "was found. all to high accuracy.

In this algorithm it is assumed that the walker is able $10 "$ look" at a neighboring slic before deciding to mov to tt. In this cose the walker ir cal!ed a "my opic" ant [ +$]$. If the "alker does nol have this abillt! vi! is a "blınd" ant l. then it mus! now e 0 e ery stle in the perımiter. and the walk of fig. Ib results. Here a acant sile rotates the walk by $\pi$. while an occupied site rotates it by $-\pi / 4$. and evidently this is a walk of the Gunn-Orturio type.

The basic idea behind the walk of iig. Ib can be used to define a "generic" walk ior an! site-percolation problem: the vacant sites send the walker back ( $\Delta \theta=\pi)$ while an occupied site rotates the walk to the nevt direction of the lattice. ( I arbitrarily use negative r? to define the newt direction here.) A similar "alis along the cacant sties can be made by going to the dual lattice and ee ersing the roles of tho stles

In fig. Io the generic walk that joins the vacant sites of ihis same cluster is shown. Th: r acant stle! satisly the connecurity of the dual lattice. which in thus case is the square lattice wliti nearest-neighbor anu nexancarest-nel=uboi communication.
Inspection of fig, Ib and Ic show's that many of the steps are redundant in that a walker sometimes goes to a ste that is guarranteed to be of a certain state by virtuc of the walker's previous position. In fig. Id a simpler, more efficient walk that visits both the occupied and vacant sites of a perimeter is shown. In this walk. the occupied sites rotate the walk to the first vertical or horizontal direction to the right. and the vacant sites rotate it to the first diagonal direction to the left. The angles of rotation are titus not fixed but either $\pm \pi / 2$ or $\pm 3 \pi / 4$ dependino upon the direction from which the site is approached. The asymmetry between the occupied and vacant sites reflects the different nature of these two sites on this lattice.

\section{Bond percolat ion on a square lattice}

A grsal variety of HGWs for bond percolation on a square lattice have been found, and 1 will briefly describe them here.

In fig. 2a a bond-percolation cluster with five orcupied bonds (solid lines) and ien vacant bonds (shaded lines) is shown. The arrow's follow a stepby-step path from bond center to bond center that Iraces out the boundary of this cluster.

In tig. $2 b$ the same process is shown on the equir. alent covering site-percolation lattice, where the sites are placed at the centers of the bonds and each site is connected to six other sites. The path of the connected arrows is precisely the walk of Gunn and Oituño [ 18$]$ in a sy'stem containing sites that rotate the walk by either $-\pi / 2$ or $\pi / 2$. corresponding to the occupied bonds and the vacant bonds, respectively. In conirast, the generic walk for this system is shown in fig. 2c, which is evidently more complicated. A genoric walk can also be construcieut that sieps from vacant stle to vacrnt site, analogous to fig. Ic. Notice that in rig. ab the ualker checks only the diagonals of the lattice.

Manna and Guttmann [26́] have pointed out that the paths of connected arrow's in nig. 2a or 2b are kinetk growth rauls (KGTs) [27]. also called grou ing self-ar viding iralls $(354 T s)[2 S]$. on the directed 


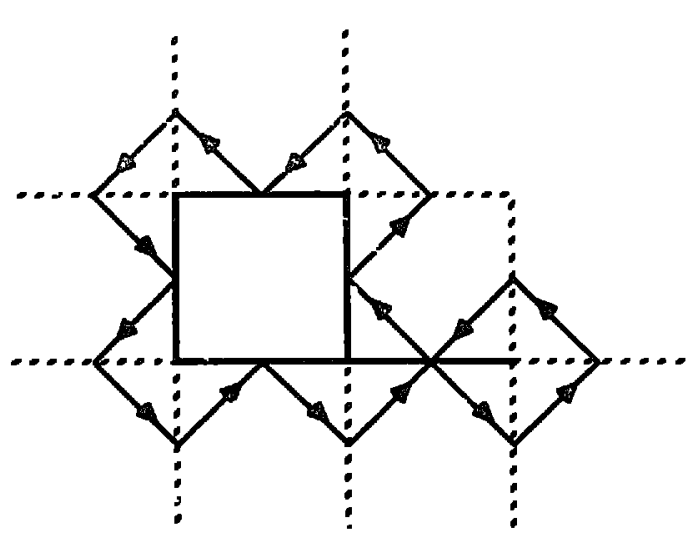

a
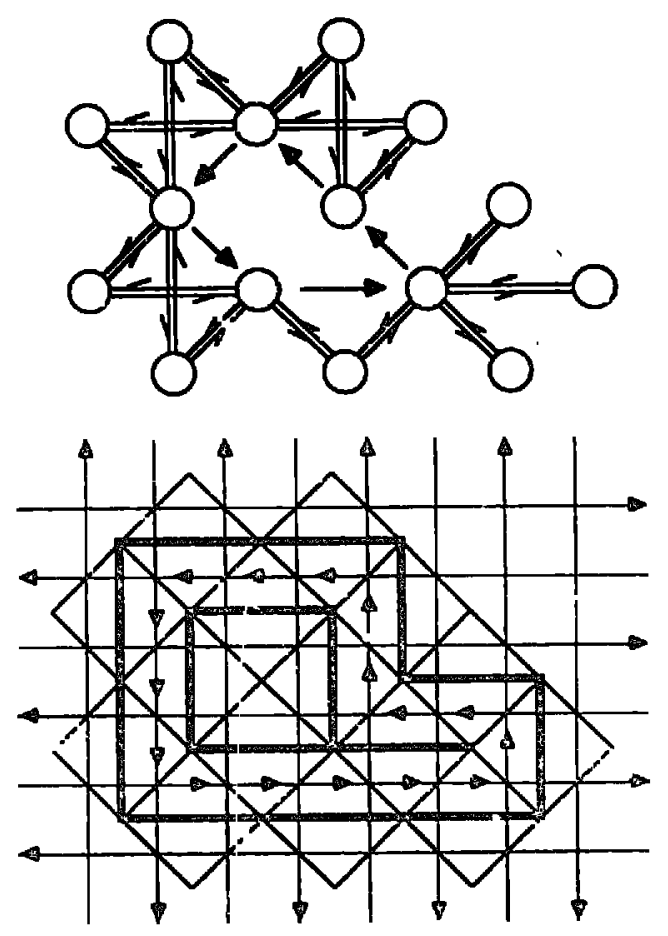

L-lattice. in which . $n$ each site there is a pair of arrows pointing in and a pair pointıng out. A KGT is a kinetic walk on a latsice that can visit each site without restriction but exch bond only' once. On the Llattice. the KGT is adtomatically a SKW because It neser gets trapped except io close.

In fig. 2d the random uling model of Rous et al. [19] is shown. In this model, the we tules shown in the upper right-hand corner oi that figure are randomly placed with equal probability on a square latlice (rotated by $\pi / 2$ here) and connecied paths are formed. The tiles ev identiy have the effect of rotating the direction of the walk as in lig. 2b. and thus this process is equi alen: is the bond HGW', as shown by<smiles>[As]</smiles>

b
C

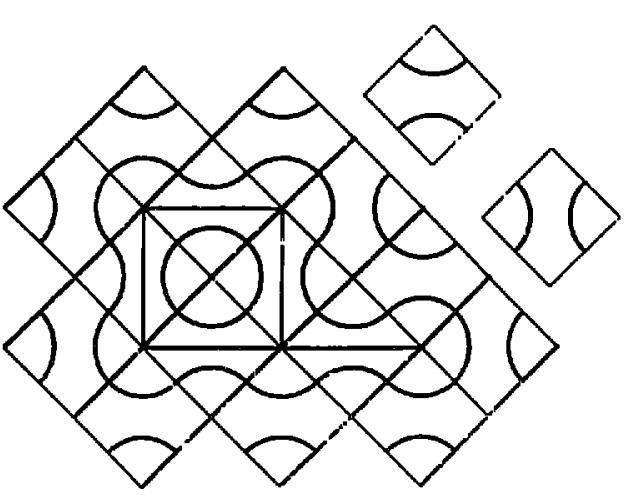

Fig. 2. Bond periolation on a sinuare latice la a duster with arrows showing an cisernal perincter path iqualent win:

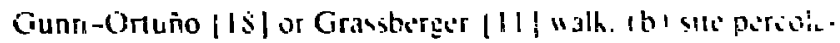

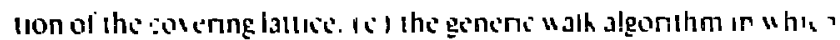

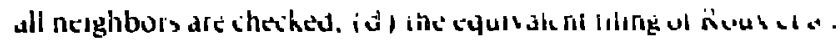
[19]. and ( 2 ) the cluster placed on a Manhation latnice on whic) the perımeter is simply a KGW [25.26].

Duplanier [29]. and Manna and Guttmann [20]. Note that the two tiles do not correspond directly to occupied and vacant siles, however. li one thinks of the lattice as being a checkerboard. then the occupted sites $w$ ill correspond to thles of one type on the w bite squares but the tules of the oppostic lipe an blash squares [29]. Reus et al. [19] always hound crilsa!-

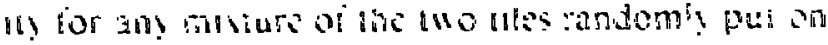

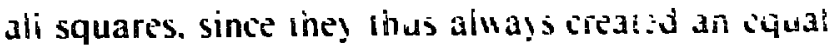
number of vacant and occupied bonds thowever. placed with a spatial bias row ards diflerent colors on the checkerboard).

In fig. 2e the cluster is placed on an underlyng Nianharan latuce with hali the latuce spacing of the 
percolation latice. The arrows of the walk around the cluster are seen to obey the resirictions of this lattice. as shown by Bradley [25] and by Manna and Guttmann [26]. Each step on this lattice corresponds to either a cut of a vacant bond or a step parallel to an occupied bond. Because of the properties of the Manhattan lattice at each step there are two possible directions to continue. The walk is a simple kinetic growth walk ( KGW). A KGW is a walk which steps with equal probability to any neighbonng site that was not previousl'; visited [30-32]. On the Manhattan lattice. the $\mathrm{K}(\mathrm{jW}$ is therefore a SKW [ 25,26].

In sirmmary the fnllowing walks are HGWs for bond percolation on a square latice:

(1) The generic walk on the covering site lattice. which can be constructed to step between eithc- the occupied (fig. $2 \mathrm{c}$ ) or vacant sites.

(2) The paths on the $\pm \pi / 2$ model of Gunn and Ortuño [18].

(3) Sull percolation on the random tiling of Roux et al. [19].

14 ) The KGT (or GSATI on an L-latuce [26].

151 The KGW' on a Manhattan lautuce [25.26].

\section{Discussion}

Thus, we have seen that many walk-forming processes. which have mostly arisen independentl: from a 1 ariety of problems. are in fact different forms of HGII. This paper has been mainly a pedagogical re$\checkmark$ iew. alihougin the "alk of nig. $1 \mathrm{~d}$ is a new and efficent Hisl for siis percolation hulls. A tuling procedure to generate threse graths can also be given. although it is not as el gant as the culing for bond percolation. This ualk can also be generalized for sitehond percolaiton.

The perimeters considered here are related. but not ident!cal, 10 the accessible perimeter introduced by Grossmon and tharon! [22]. The accessible perim. ete, is the external perimeter of a cluster that can be probed by a particle of a gisen size moring along a path of nearesi-neighbor vacant sites from infintty. then thes particle is sufficiently large idepending upon the lattice). the invaginations of the cluster are cut off and the remaining hull is found to have a fractal dimension of $\approx 4 / 3$ rather than the $7 / 4$ of the complete perimeter, and thus of a different universality class. Note that for a perimeter generated by the HGW, we can define the accessible perimeter as all sites that can be reached from infinity without crossing any path of tie nGW, which is thus a definition independent of the size of a probe particle and the type of lattice. To generate the accessible perimeter by a walk process. one must first generate the complete perimeter in the usual way with a HGW, and then carry out another scouting walk around the perımeter to identify the "hull" of the hull [21.22]. In fact, this new hull is more in the spirit of Mandelbrot's original definition of the word than the more common usage as iny perimeter, and furthermore the fractal dimension $(4 / 3)$ is exactly identical to the value conjectured by Mandelbrot [ 1 ] for the Brown hull (which is the accessible perimeter of the Brown trail ), based upon the value for the self-avoiding random walk. There is no lucal ualk that can generate the accessible perimeter from scratch. which is pcrhaps celated to its being of a different universality class.

The connections between HGWs and KGWs. SKWs. IGSAWs, etc. aie numerous but their exact nature is dependent upon the specific lattice and sysIem being considered. In many cases. such walks are not precis.ly HGWs but of the same universaltty class. One examp!e is the IGS.AW of Kremer and Lyklema [17] on a sciuare lattice. While the growing end of the IGSAW never gets trapped. the non-growing end easıly does [17] In contrast. for the IGSAW (or the SK( $W$ ) on the honeycomb lattice introduced in ref. [12] neither end will get rraoped and the walk will always ev entually close, v:cause it is a HGW:

It is useful to make this distinction between "bcing of the same unwersaluty class as a $\mathrm{HGW}^{\prime \prime}$ and "being a "ype of a HGW". which is a stronger statement. As we have seen. there are many random walks that are a lype of HGW, which means that any results of their simulation apply equall! to percolation hulls. This can lead to ambiguty when refering to such walks - do 
they represent SKWs. or percolation hulls [23]? The answer depends upon the lattice - for site percolation on the triangular lattice the HGWs are both, while for the square lattice the HGW (fig. 1) is somewhat different than the SKW or IGSAW. I would also like to point out that in ref. [23], the very extensive simulations of ref. [10], which gave $1 / D=4 / 7 \pm 0.0005$. were misquoted to a much lower precision.

The various considerations given here for the square lattice can be applied to the many other twodimensional lattices, including directed ones, to yield a great variety of interesting HGW's.

\section{Acknow ledgements}

I thank S. Manna and A. Guttmann, B. Duplantier and $H$. Saleur, and $R$. Bradley for sending preprints of their work. I also w ish to acknowledge support from the National Science Foundation gran: No. DMR8619731 .

\section{References}

[1/B.B. Miandelbrot. The Fratal Gionkery d' Nature (Freeman. San Francisco 1983) pp. 126-130, 132.242.

[2] H... Herrmann. Phys. Rep. 136 ( 1986 ) 153.

[3] T. Vlisek. Fractal Growth Phenomena (World Scienutic. Singapore. 1989).

[4] H E. Stanle!. In: On Growih and Form. H.E. Stanles and N. Ostrousks. eds I Nijhoft. The Hague. 19561.

[5] P.G. de Gennes. P Lafore and J.P. Mlllot J. Phy s. Chem. Soltds: 111959$) 105$.
|6| M.E. Fisher and I Essam. I Math Phss 2 1 lyol I ouw [7] G.R. Reich and P.L. Leath, J. Phys. $[$ H (iyio) iljj. tui i.

[8] B. Sapoval. M. Rosso and J.-F. Couyer. J. Phys (Yaris) tó (1985) L149.

[9] R. Voss. J. Phys. A 17 , 1984 ) L373.

[10] R.M. Ziff, Phys. Rev. Lett. 56 (1986) 545.

|11 | P. Grassberger. J. Phys. A 19 ( 1986 ) 2675.

[12] 4. Weinnb and S.A. Trugman. Phı̣s Rev. B 31 (1985) 2993.

[13] H. Saleur and B. Duplanıer. Phỵs. Rax Letı. 58 (1987) 2325.

[14] A. Bunde and J.-F. Gouyet. J. Phys. + I8 (1985) LI85.

[ 5 ] R.M. ZıIT. P.T. Cummıngs and G. Stell. J. Phys. A 17 (1984) 3009.

[16] R.M ZirT. presented al Stalisucal Mechanics Meeung at Rutgers. May. 1982. J. Stal. Phys 28(1982) 8.38.

[17] K. Kremer and J.W Lyklema. Phys. Reu Lell $5+$ (1955) 267: J. Phys. A 18 i1985) I5। s.

[18] J.M.F. Gunn and M. Oriuño. J. Phys. +18 (1985) L1095.

[19] S. Roux. E Guson and D Sometle. J Pḩs. + 21 (1985) L475.

[20] R.M. Ziff and B. Saporal. J. Phis $+1 S(1950)$ LI I64

[21 IS.S. Manna. J Phys + 22 $119381+1\}$

[22! T Giossman and t. tharm! J Ph!s 119,1430 ) L 7ti. $20 ̈(1957) L 1193$

1231 + Conglo N Jan. I Mand and HE Sianks Ph! R.: B 35,$19871301 ;$

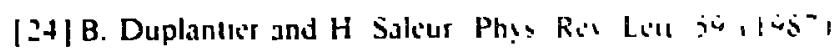

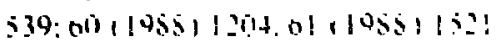

[2S! K.M. Bradle! Phos RL + is q fund ? is

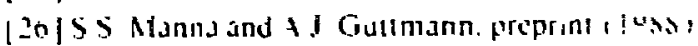

[2]] t. Malakis. J Phys. A o lyij I loos.

[28 ] J.W. Lyklema. J. Phys. A I8 (1985) Lol?

[39] B. Duplantıer. J. Phys. $+2111980,3464$

[30] J.W'. Lyklems and K. Kremer. J Phus +1 , 1954 L64l. $19(1986) 279$

[31] I. Majld. N. Jan. a Coniglio and H E Siantes. Ph!s. Re Lell. $52(195+1) 1257$.

132IS Hemmer and PC Henmer. I Chem Phys S! 1 49t, ist 